\title{
C3-BIOECONOMY
}

Circular and Sustainable Bioeconomy

\section{Bioeconomía, estrategias e impacto}

Alfredo Aguilar ${ }^{1}$

Autor de Correspondencia: alfredo.aguilar@efbiotechnology.org

\section{Resumen:}

La bioeconomía tiene quince años de existencia y en esos años se ha extendido por más de cincuenta países y regiones de todo el mundo. Surgió como medio de buscar una alternativa a la economía basada exclusivamente en la explotación del petróleo y de otros recursos fósiles. La bioeconomía se adapta por su naturaleza al clima, desarrollo agrícola, industrial y socioeconómico de un país o región y a su entorno político. En la actualidad, hay un gran consenso a nivel global sobre el hecho de que la bioeconomía ha de ser circular, sostenible, usar materias primas renovables y aceptar los límites ecológicos del planeta. La experiencia de estos años permite reflexionar sobre la forma de incrementar el impacto de la bioeconomía aprendiendo de aquellas experiencias exitosas. En el presente artículo se postulan los siguientes temas: necesidad de una coherencia entre la bioeconomía y las otras políticas; generar un amplio consenso social y político; las estrategias y los planes de acción deben de ser inclusivos y combinar estrategias con acciones concretas. Se avanza asimismo unas consideraciones entre los conceptos de bioeconomía, sostenibilidad y biodiplomacia.

Palabras clave: bioeconomy, sostenibilidad, biodiplomacia, recursos naturales, economía circular

\section{Bioeconomy, strategies and impact}

\author{
Alfredo Aguilar ${ }^{1}$
}

\section{Abstract:}

Bioeconomy has been in existence for fifteen years and in those years, it has spread to more than fifty countries and regions around the world. It emerged as a means of seeking an alternative to an economy based exclusively on the exploitation of oil and other fossil resources. Each specific bioeconomy is adapted by its nature to the climate, agricultural, industrial and socioeconomic development of a country or region and to its political environment. At present, there is a great consensus at a global level that the bioeconomy must be circular, sustainable, use renewable raw materials and accept the ecological limits of the planet. The experience of these years allows us to reflect on how to increase the impact of the bioeconomy by learning from those successful experiences. In this article the following themes are proposed: need for coherence between the bioeconomy and other policies; generate a broad social and political consensus; strategies and

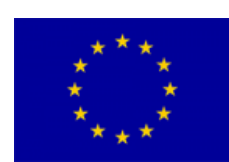

UNIÓN EUROPEA PROYECTO COFINANCIADO POR EL FONDO EUROPEO DE
DESARROLLO REGIONAL
(FEDER) Una manera de hacer Europa

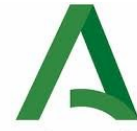

Junta de Andalucía
Andalucía

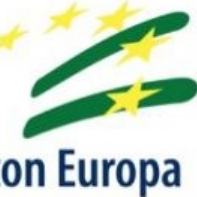


action plans must be inclusive and combine strategies with concrete actions. It also advances some considerations between the concepts of bioeconomy, sustainability and biodiplomacy.

Key Words: Bioeconomy, sustainability, biodiplomacy, natural resources, circular economy

1 Working Group Bioeconomy, ESAB, European Society for Applied Biocatalysis, Frankfurt Am Main, Alemania; Ex - Jefe de Unidad Biotecnologías, (Comisión Europea), Bruselas (Bélgica), alfredo.aguilar@efbiotechnology.org.

\section{RAZONES PARA UNA BIOECONOMÍA}

Las razones que movieron a las dos grandes regiones del planeta, la Unión Europea (UE) y los EE. UU., en la primera década del siglo XXI, a desarrollar iniciativas a favor de una bioeconomía fueron diferentes, y en consecuencia sus objetivos, estrategias e iniciativas también lo fueron. La UE imprimió al concepto de bioeconomía, una sólida base científica y técnica, con objeto de desarrollar los conocimientos científicos para hacer un planeta más "verde" y menos dependiente del petróleo. Así, el concepto de Bioeconomía basada en el conocimiento o KBBE, por sus siglas en inglés fue desarrollado en 2005 por la Comisión Europea y dos años más tarde por Alemania que ocupaba en aquellos momentos la presidencia de la UE. Ése fue el primer paso decisivo para definir un marco de actuación y procurar que otras políticas como la agrícola, industrial o de medio ambiente, entre otras convergieran hacia el nuevo concepto de bioeconomía. Unos años más tarde, en 2012 la UE adoptó la Estrategia en Bioeconomía (European Commission, 2012). Es importante señalar aquí, que ninguno de estos documentos surgió de la nada o de la improvisación. Hubieron de pasar diez años entre la adopción de una estrategia europea sobre Biotecnología (European Commission, 2002) y la estrategia en bioeconomía y treinta años desde el primer programa europeo de biotecnología y la adopción de la Estrategia de Bioeconomía (Patermann \& Aguilar, 2018) (Aguilar et al, 2013). Es importante resaltar este aspecto ya que iniciativas de esta envergadura no solo requieren decisión e iniciativas políticas claras, también necesitan que exista una variedad de instrumentos de gestión, madurez en las 
políticas sectoriales y un gran consenso socioeconómico y político. Sin estos prerrequisitos es muy probable que cualquier iniciativa, por muy loable que sea, esté condenada al fracaso por la dificultad y ausencia de seguimiento o de implementación.

Por su parte, los EE. UU. basaron sU estrategia en garantizar en la medida de lo posible el suministro energético en un momento en que las relaciones políticas con varios países exportadores de petróleo pasaban por una tensa situación (National Bioeconomy Blueprint, 2012). Los objetivos principales eran estimular el desarrollo económico basado en la innovación y acercar esas innovaciones al mercado de forma que permitieran a EE. UU. seguir manteniendo el liderazgo en temas de innovación.

En los años posteriores al 2012 más de 50 países de todos los continentes han desarrollado iniciativas o estrategias en relación con la bioeconomía. Este artículo no permite hacer un análisis, siquiera superficial, de todas y cada una de esas iniciativas y estrategias; El lector interesado puede consultar las referencias publicadas por la Oficina del Consejo de Bioeconomía Alemana donde se describe las diferentes iniciativas que existen en la actualidad (Bioeconomy Policies Part I, Part II \& Part III). Muchas de estas iniciativas se inspiraron de las estrategias de Alemania, país pionero en Bioeconomía, de la UE y de los EE. UU. Quizá tan sorprendente como el número de estrategias nacionales y muchas más de carácter regional, es la diversidad de estas estrategias. No existe, contrariamente, a lo que algunos adelantaban de una forma demasiado reduccionista, una sola bioeconomía, sino una gran diversidad de bioeconomías adaptadas cada una al clima específico, a la agricultura, al desarrollo socioeconómico y científico-técnico, etc. Sin embargo, es necesario insistir en el hecho fundamental que deben de reunir todas las bioeconomías, que es el desarrollo de un sistema socioeconómico basado en el uso sostenible de los recursos biológicos respetando, además, los límites ecológicos del planeta. 
Si la base del conocimiento fue el punto de partida de la bioeconomía de la UE, este abordaje se ha complementado a lo largo de los años con una visión más holística: hacer compatible y sinérgica la bioeconomía con otras políticas comunitarias, a la vez que se busca un apoyo decidido y claro de la sociedad. La bioeconomía necesita para tener éxito, guardar un delicado equilibrio entre el empuje de la ciencia y de la tecnología, apoyado principalmente por el sector público y un tirón del mercado y de la sociedad, estimulado a su vez por los sectores agrícolas, industriales y financieros y dentro de un marco de políticas coherentes. En cada una de estas etapas, la consulta, participación y apoyo de la sociedad en su conjunto en la preparación y toma de decisiones es absolutamente imprescindible. En este sentido, la UE adaptó en 2018 su Estrategia de Bioeconomía del 2012 en la que aspectos como sostenibilidad, economía circular, así como la imperiosa necesidad de conocer los límites ecológicos de la bioeconomía pasaron a formar parte de forma inseparable del concepto de bioeconomía (European Commission, 2018).

En los últimos años ha surgido un amplio movimiento social, principalmente entre los jóvenes de todo el mundo, reclamando a los poderes públicos un uso más racional de los recursos biológicos a la vez que exigen cambiar un sistema productivo desfasado que hipoteca el desarrollo de las generaciones futuras. Hay iniciativas incipientes, tanto de instituciones como de particulares tratando de que iniciáticas tales como el calentamiento global, la seguridad alimentaria, el incremento de población y la preservación del medio ambiente, incluido el de los océanos sean abordados desde una perspectiva global. Así, primarían los intereses de la humanidad en su conjunto sobre los legítimos, pero más limitados de las prioridades nacionales o regionales (Marvik \& Philp, 2020) (Aguilar \& Patermann, 2020). En este sentido se ha propuesto la biodiplomacia como un nuevo instrumento adicional a los ya existentes para una gestión global y eficaz de los recursos biológicos y de los grandes retos a los 
que se enfrenta el planeta, como cambio climático, seguridad alimentaria e incremento de la población humana, entre otros (Aguilar \& Patermann, 2020).

Muy recientemente, el IACGB, International Advisory Council on Global Bioeconomy, ha publicado en la última Cumbre Global de Bioeconomía 2020, GBS2020, su cuarto informe en el que hace un análisis crítico muy interesante de la evolución de las políticas e iniciativas en los distintos países del mundo en relación con la bioeconomía, así como su evolución en los últimos años (International Advisory Council on Global Bioeconomy, IACGB, 2020). De particular interés en este contexto es también el Comuniqué o declaración final del GBS2020, que orienta sobre las distintas sensibilidades y los desafíos globales a los que se enfrenta la bioeconomía (IACGB, 2020).

Es claro que la bioeconomía está llamada a desarrollar un papel importante en la agenda política y social actual del futuro a corto y medio plazo. Sin embargo, su grado de implicación y de consideración política y social dependerá en gran medida en la forma en la que la bioeconomía, o bioeconomías respondan a la demanda social de abordar y resolver, o al menos mitigar o adaptarse a los retos globales a los que la humanidad se viene enfrentando. Es importante recordar una vez más que la bioeconomía no es una disciplina científica, ni tampoco una tecnología, ni incluso un sector industrial. El concepto de la bioeconomía se genera por la integración de todos estas disciplinas, tecnologías y sectores agrícolas e industriales, integrados en un contexto socioeconómico y con el objetivo políico de gestionar un uso de los recursos naturales de forma sostenible y económicamente viable (Aguilar et al, 2019).

\section{BIOECONOMÍA Y OTRAS POLÍTICAS}

Uno de los aspectos más importantes en el desarrollo de una estrategia sobre bioeconomía es el de asegurar una coherencia con otras políticas e iniciativas, tanto nacionales o regionales, así como el asegurarse el apoyo de los sectores 
socioeconómicos. Nunca se insistirá suficientemente en la imperiosa necesidad de implicar a la sociedad en su conjunto en todas y cada una de las etapas, tanto de la preparación, como en su implementación. Siendo la bioeconomía una actividad con vocación de tener un impacto socioeconómico positivo, es preciso que su implementación sea armónica, coherente y sinérgica con el conjunto de las otras políticas ya existentes.

Es de particular importancia garantizar la máxima conexión e interacción con las políticas llamémoslas tradicionales, como la agrícola, industrial, o medioambiental. Bastantes de los beneficios de la bioeconomía, como nuevos puestos de trabajo, nuevas tecnologías, impacto positivo sobre el medio ambiente, entre otras, se formarán en las interfaces de esas políticas con la bioeconomía. Es por tanto crítico el cuidar esas interacciones y promover diálogos, asistencia y atención con vistas a permitir que tanto las políticas tradicionales como la bioeconomía salgan reforzadas de esas interacciones. A continuación, se describen muy sucintamente algunas de las iniciativas de la Unión Europea, con las que ha habido interacciones mutuas positivas entre bioeconomía y otras políticas. Dada la complejidad de las múltiples interacciones, solo se mencionarán aquellas iniciáticas de la UE cercanas a la investigación y a la innovación:

- Food 2030. En esta iniciativa del 2015, la UE prioriza la seguridad alimentaria en Alimentación y Nutrición mediante una producción de alimentos sostenible y saludables y accesibles para toda la población (European Commission, 2015).

- Blue Growth. Esta iniciativa trata de ser una respuesta de la UE a la Agenda de Desarrollo Sostenible de Naciones Unidas, y en particular a su objetivo $\mathrm{n}^{\circ}$ 14: "Conservar y utilizar sosteniblemente los océanos, los mares y los recursos marinos" (European Commission, 2019).

- Bio-Based Products and Processing. Esta iniciativa conjunta de la Unión Europea y de las bioindustrias europeas tiene como objetivo promover y 
desarrollar nuevas tecnologías que sirvan para la transformación sostenible de los recursos biológicos renovables (Reglamento (UE) n 560/2014) (Mengal et al, 2018) (Ruíz Sierra et al, 2020).

- International Bioeconomy Forum. El objetivo de esta iniciativa es asegurarse que la bioeconomía reciba la importancia que merece a escala internacional y en concertación con iniciativas globales en marcha como COP21, los ODS, objetivos de desarrollo sostenibles, economía circular, seguridad alimentaria y al mismo tiempo promover investigación e innovación a nivel internacional para contribuir a los objetivos políticos de la bioeconomía (European Commission, 2020).

Recientemente, las autoridades de la UE han adoptado la iniciativa del Pacto Verde Europeo. El ambicioso Pacto Verde, de un gran calado políicico, pretende que Europa se esfuerce y consiga de cara al año 2050 ser el primer continente climáticamente neutro. El Pacto Verde conlleva una hoja de ruta para dotar a la UE de una economía sostenible y próspera. La realización de este objetivo exigirá la transformación de los retos climáticos y medioambientales en oportunidades en todos los ámbitos de nuestro entorno de forma que se logre una transición justa e integradora para todos (El Pacto Verde Europeo, 2019). El Pacto Verde Europeo establece un plan de acción para impulsar un uso eficiente de los recursos mediante el paso a una economía limpia y circular, así como restaurar la biodiversidad y reducir la contaminación ambiental. El Pacto Verde describe igualmente las inversiones necesarias y las herramientas de financiación disponibles y explica cómo garantizar una transición justa e inclusiva. Para alcanzar este ambicioso objetivo, será necesario actuar en todos los sectores de nuestra economía a través de todos y cada uno de los países y regiones de la UE. En particular, mediante la inversión en tecnologías respetuosas con el medio ambiente; en el apoyo a la industria innovadora; en el desarrollo y despliegue de sistemas de transporte público y privado más limpios, más baratos y sanos para los usuarios, los seres vivos y el 
medioambiente; contribuyendo a descarbonizar el sector de la energía y garantizar que los edificios sean más eficientes desde el punto de vista energético. Por último, la UE desea tomar el liderazgo a nivel internacional para colaborar con otros socios regionales e internacionales para mejorar las normas medioambientales mundiales.

La UE también proporcionará apoyo financiero y asistencia técnica para ayudar a las personas, las empresas y las regiones más afectadas por la transición hacia la economía verde. Esto se denomina el Mecanismo para una Transición Justa. Ayudará a movilizar al menos 100.000 millones de euros durante el período 2021-2027 en las regiones más afectadas.

El Pacto Verde Europeo tiene bastantes elementos convergentes con la Estrategia Europea de la Bioeconomía, aunque esas sinergias no se hayan evidenciado en los documentos iniciales del Pacto Verde. Es de esperar que los desarrollos ulteriores de las actuaciones y programas del Pacto Verde, así como de la Estrategia Europea de Bioeconomía pongan en evidencia de una forma más visible una mayor sinergia y convergencia en sus acciones.

Más allá de la UE, representantes de la Organización para la Cooperación y Desarrollo Económico, OCDE y de Noruega, han señalado la absoluta necesidad de integrar el concepto de bioeconomía en una perspectiva más amplia relativa a una estrategia renovable del ciclo del carbono que integre la producción de biomasa con el ciclo industrial del carbono (11). Esta línea de pensamiento coincide con la postulada previamente por Aguilar y Patermann (2020), en la que señalan la urgente necesidad de abordar de una forma global los grandes retos o desafíos que tiene la humanidad en este momento y que de no hacer nada, se agravarán irreversiblemente. Estos autores postulan que no es posible abordar de una forma sectorial o parcelada los grandes retos del planeta, y que como se ha mencionado anteriormente son bien conocidos: cambio climático, seguridad alimentaria, incremento de la población humana, entre otros. Cada uno de estos desafíos tiene innumerables consecuencias en 
distintas áreas y sectores de nuestra vida. Además, los desafíos a los que nos enfrentamos están íntimamente ligados entre sí. Es utópico pensar que el único efecto del cambio climático sea el calentamiento global y que, luchando contra el calentamiento global desde una óptica reduccionista, se resolverá el problema. El cambio climático tendrá, de hecho, está teniendo ya efectos devastadores en la agricultura en determinados países, en la trasmisión de zoonosis y otras enfermedades y en consecuencia provocará migraciones y desplazamientos de poblaciones humanas de dimensiones y consecuencias insospechadas, incluso en términos de seguridad y conflictos armados. Se requiere, por tanto, un abordaje holístico, guiado por Naciones Unidas y apoyado por las regiones y países comprometidos con estas políticas sostenibles de manera que sirva de estímulo y revulsivo de forma que otros países se incorporen a este esfuerzo colectivo. Para que este proceso tenga éxito es necesario reemplazar, al menos para la gestión de los recursos biológicos del planeta, la diplomacia clásica de los países, en el que prima el interés nacional frente al colectivo, por una biodiplomacia donde primen los intereses globales de la humanidad. Nuestra supervivencia como especie, al menos en la forma en que la conocemos actualmente, dependerá de la forma en que los líderes políticos tomen conciencia de la dimensión de los problemas a los que nos enfrentamos la humanidad como especie, y amplíen su horizonte y su campo de visión política. Los lectores interesados pueden consultar en la referencia $n^{\circ}$ 12, un desarrollo más elaborado de las interacciones entre bioeconomía, biodiplomacia y los desafíos globales del planeta relacionados con la biosfera.

El Club de Roma ha publicado recientemente un estudio, complementario al anterior en el que se aborda en profundidad las consecuencias catastróficas que conllevaría la continuación de los modelos económicos de la actualidad, insostenibles y depredatorios con el medio ambiente y con los límites ecológicos del planeta (Ulrich Von Weizsäker \& Wijkman, 2018). Dicha obra analiza igualmente alguna de las nuevas teorías económicas que se han venido 
desarrollando en los últimos decenios que promueven un desarrollo económico y una explotación de los recursos biológicos sostenible que garantice los recursos existentes para las generaciones futuras.

El concepto de bioeconomía ha evolucionado enormemente desde su aparición hace quince años. En 2009 la OCDE dio a la bioeconomía una definición bastante restrictiva: "La bioeconomía se ocupa de las actividades económicas relativas a la invención, desarrollo producción y uso de productos y procesos biológicos" (The Bioeconomy to 2030, 2009). Es decir, la bioeconomía sería una economía como las demás, pero basada en productos de origen biológico. La UE en su estrategia Europea de la Bioeconomía de 2012 ya mencionaba que, "La bioeconomía se ocupa de aquellas partes de la economía que usan recursos biológicos renovables procedentes de la tierra y del mar, - tales como cultivos, bosques, peces, animales y microorganismos para producir alimentos, materiales y energía." (European Commission, 2012). En este caso se introducía el concepto de recursos renovables. Por su parte, la Cumbre Global de Bioeconomía de 2015 o GBS por sus siglas en inglés, introdujo en la definición de bioeconomía el concepto de sostenibilidad: "La bioeconomía consiste en la producción y utilización de recursos biológicos basados en el conocimiento, procesos y principios biológicos innovadores para proporcionar bienes y servicios de manera sostenible en todos los sectores económicos" (Communiqué Global Bioeconomy Summit, 2015). Pero no es finalmente hasta 2018, cuando la UE, en su revisión de la Estrategia de Bioeconomía, que los conceptos de sostenibilidad, economía circular, y sobre todo la necesidad de conocer y comprender los límites ecológicos de la bioeconomía, entran a formar parte integral del concepto de la bioeconomía (European Commission, 2018).

\section{IMPACTO DE LA BIOECONOMÍA: LECCIONES PARA EL FUTURO}

Hasta muy recientemente la bioeconomía, o mejor, las distintas estrategias de las bioeconomías se han venido construyendo de forma "top down" o de 
arriba abajo. Generalmente, desde círculos políticos, basados en los sectores técnicos y científicos y basadas en el conocimiento científico y tecnológico de los seres vivos. Estos abordajes han servido durante algo más de una década para construir el andamiaje de las acciones a desarrollar para poner en práctica las bioeconomías. Dado el alto nivel de especialización de algunos contenidos de la bioeconomía, estos abordajes han sido, posiblemente y a pesar de algunas críticas, los únicos posibles en las circunstancias en que se desarrollaron dichas estrategias. Es necesario reconocer que un buen número de esas estrategias han sido muy exitosas y continúan desarrollando acciones y proyectos concretos con impacto visible para la sociedad y el tejido socioeconómico. Sin embargo, las estrategias de un buen número de países y regiones que se construyeron sin esos consensos adolecen frecuentemente de un limitado impacto en sus sociedades respectivas.

Un análisis exhaustivo de las causas de las razones por las que un cierto número de estrategias e iniciáticas en bioeconomía no se han trasladado a los tejidos sociales y económicos requeriría un análisis bastante más profundo de lo que permite este artículo. No obstante, a continuación, se esbozan brevemente algunas de las razones que podrían haber hecho naufragar la puesta en práctica de, por otro lado, buenas estrategias de bioeconomía.

Necesidad de un amplio consenso social y político. La primera ha sido para algunos líderes e instituciones, el pensar que la elaboración de una estrategia era un fin en sí mismo, y no el principio de un largo proceso que culminaría con la incorporación en la sociedad de buenas prácticas de bioeconomía. Algunas de estas estrategias se han limitado a ser una declaración política de intenciones y les ha faltado el desarrollo de un plan de trabajo y un plan de seguimiento. Frecuentemente, la bioeconomía se ha visto por bastantes líderes como iniciativas de partido, dando lugar a que cambios en el color político del gobierno o de la administración en cuestión, se vete o en el mejor de los casos se ignore cualquier iniciativa en bioeconomía de las administraciones anteriores. 
En las estrategias que se han convertido en éxitos de implementación y aceptación social se observa en todas y cada una de las etapas de preparación de estrategias, como de ejecución de los planes de acción, un amplio dialogo con los actores científicos, técnicos, industriales y sociales, así como la búsqueda de consenso con las otras fuerzas políticas. Este proceso alarga, sin duda, la elaboración de la estrategia, pero permite recoger sensibilidades y aspectos específicos importantes para determinados sectores. Permite igualmente, el asumir por la mayoría de la sociedad y sus representantes la corresponsabilidad en la planificación y gestión de las estrategias y sus mecanismos de acción.

Las estrategias y sus planes de acción deben de ser inclusivos. Un factor crítico para una amplia aceptación social es prestar una atención particular para no dejar a ningún sector social fuera de los aspectos positivos o beneficiosos de la bioeconomía. Una estrategia que apoye y beneficie a un determinado sector a costa de otro, o que los posibles beneficios no redunden en el conjunto de los actores sociales tendrá probablemente una vida muy corta. Un ejemplo paradigmático de esto es el desarrollo inicial de los OGM, organismos modificados genéticamente, allá por los años ochenta del siglo pasado en el que los beneficios estaban concebidos casi exclusivamente para los productores de semillas y los agricultores, y en los que la opinión pública no tuvo apenas voz. El resultado es conocido por todos y de esa lección se debería tomar nota de una vez por todas que en los temas en los que interaccionen aspectos científico-técnicos, con un amplio impacto social en sectores muy sensibles tales como alimentación, medio ambiente, salud, etc., es absolutamente imprescindible obtener un amplio consenso social. Este consenso social se basa en varios factores: una información fehaciente y veraz; un dialogo sin líneas rojas en el que intervengan todos los actores y sectores con intereses legítimos en el tema y, por último, generar un proceso de codecisión y co-gobernanza que favorezca la corresponsabilidad en la toma de decisiones. 
La evidencia científica y la fiabilidad tecnológica son elementos absolutamente imprescindibles, pero es crítico para una implementación exitosa de una estrategia en bioeconomía generar un clima de confianza entre los distintos actores en el que se comparta un objetivo común.

Combinar estrategias con acciones concretas. La bioeconomía no se desarrolla solamente con la elaboración de ambiciosas estrategias ni tampoco con la ejecución de proyectos descoordinados unos de otros en ausencia de estrategia alguna. Existen, desgraciadamente ejemplos suficientes que demuestran que estrategias concebidas y elaboradas sin un amplio diálogo y consenso social y sin un plan de acción que las acompañen, no llegan a tener apenas impacto. Por otra parte, la ejecución de proyectos y acciones sin una planificación estratégica y un seguimiento de resultados y de impacto, tiene, en general, un efecto movilizador solo temporal mientras duren las acciones y los proyectos. Es necesario, por tanto, combinar indisolublemente estrategias con acciones concretas y que generen resultados visibles. En este sentido es crítico hacer converger conceptos de alto nivel tales como:

- la preservación del capital natural de nuestro planeta, tanto biológico como no biológico;

- conectar economía y ecología;

- mantener las condiciones de sostenibilidad y habitabilidad de la biosfera.

Entre las acciones concretas y verificables se encontrarían:

- acciones muy específicas con impacto concreto y medibles sobre el terreno sobre el que se actúa;

- programas y planes de acción conducentes a la creación de empleo y a la creación de programas y planes conducentes a desarrollar un crecimiento económico sostenible. Todas estas acciones concretas deberían ser evaluadas 
por comités independientes. Este apartado se ha desarrollado recientemente en profundidad por Wohlgemuth et al (2021).

\section{BIOECONOMÍA, SOSTENIBILIDAD Y BIODIPLOMACIA}

Posiblemente la evolución más importante en el desarrollo de bioeconomía en los últimos años ha sido la incorporación del concepto de sostenibilidad en un ecosistema local y encuadrar la bioeconomía dentro de los límites ecológicos del planeta a un nivel más global. Frente a la actitud depredatoria seguida en los dos últimos siglos respecto a la extracción y consumo de los combustibles fósiles, la bioeconomía pone esos dos conceptos claves como marco de su actuación. No es posible hacer, como algunos han intentado, trasladar el modelo económico de la economía derivada de los recursos fósiles y hacer una traslación mecánica a la bioeconomía. En una reciente monografía en la que han colaborado numerosos autores de habla española se desarrollan los conceptos de bioeconomía teniendo como telón de fondo la sostenibilidad, el desarrollo de una bioeconomía circular y su relación con la sociedad en su conjunto (Aguilar et al, 2018).

Por otra parte, la bioeconomía es una actividad que por su naturaleza pivota alrededor de numerosos sectores industriales, agrícolas, científico-técnicos y por supuesto sociales sobre la que no es posible tener una visión reduccionista. En este sentido, la bioeconomía pretende contribuir, con todas sus potencialidades, pero también siendo consciente de sus limitaciones, a las discusiones que tienen lugar en la actualidad a escala global sobre los grandes retos del planeta. Recientemente se ha propuesto el desarrollo de una biodiplomacia que teniendo como base conceptual una bioeconomía circular sostenible, articule y gestiones los recursos biológicos del planeta. Esta gestión debería ser radicalmente diferente a la que se ha venido haciendo con los recursos fósiles de forma que se puedan garantizar los recursos biológicos a las generaciones futuras y garantizar la sostenibilidad de nuestro planeta (Aguilar \& Patermann, 2020). En este sentido los autores proponen, además, que los 
grandes retos del planeta, tales como seguridad alimentaria, incremento de la población, cambio climático, preservación del medio ambiente, entre otros, sean tratados de una forma integrada y gestionada por las Naciones Unidas en base de una biodiplomacia aceptada por un número importante de países.

En resumen, la bioeconomía está destinada a jugar un papel muy importante en nuestras sociedades. Para ello es necesario un cambio en el paradigma productivo y de consumo de recursos de forma que la sostenibilidad presente y futura de los recursos biológicos esté garantizada. Esta actitud nos concierne a todos, científicos, tecnólogos, agricultores, empresarios, entidades financieras y por supuesto al conjunto de la sociedad. De nuestra actitud responsable y decidida, depende el futuro de las generaciones venideras.

\section{REFERENCIAS}

AGUILAR, A., MAGNIEN, M. \& THOMAS, D. (2013). Thirty years of European biotechnology programmes: from biomolecular engineering to bioeconomy. New Biotechnology, 5, 410-425. https://doi.org/10.1016/j.nbt.2012.11.014.

AGUILAR, A. \& PATERMANN, C. (2020). Biodiplomacy, the new frontier for bioeconomy. New Biotechnology, 59, 20-25. https://doi.org/10.1016/j.nbt.2020.07.001.

AGUILAR, A., RAMÓN, D. \& EGEA, F.J. (Eds.) (2018). Bioeconomía y desarrollo sostenible. Cajamar Caja Rural. Recuperado de: https://www.publicacionescajamar.es/publicacionesperiodicas/mediterraneo-economico/mediterraneo-economico-31bioeconomia-y-desarrollo-sostenible. 
AGUILAR, A., TWARDOWSKI, T. \& WOLHGEMUTH, R. (2019). Bioeconomy for sustainable development. Biotechnology Journal, 14(8), 1800638, 1-11. https://doi.org/10.1002/biot.201800638.

Bioeconomy Policy-Synopsis and Analysis of Strategies in the G7. (2015). Berlin, Office of the German policy Council. Recuperado de: https://gbs2020.net/wp-

content/uploads/2020/04/BOER Laenderstudie 1 .pdf.

Bioeconomy Policy (Part II). Synopsis of National Strategies around the World. (2015). Berlin, Office of the German policy Council. Recuperado de: https://biooekonomierat.de/fileadmin/international/BioeconomyPolicy Part-II.pdf.

Bioeconomy Policy (Part III) Update Report of National Strategies around the World. (2018). Berlin, Office of the German policy Council. Recuperado de:

https://biooekonomierat.de/fileadmin/Publikationen/berichte/GBS 2018 _Bioeconomy-Strategies-around-the_World_Part-III.pdf.

Communiqué Global Bioeconomy Summit 2015. Making Bioeconomy work for Sustainable Development. (2015). Global Bioeconomy Summit. Recuperado de: https://gbs2015.com/fileadmin/gbs2015/Downloads/Communique_final neu.pdf.

El Pacto Verde Europeo. Comunicación de la Comisión al Parlamento Europeo, al Consejo, al Comité Económico y Social y al Comité de las Regiones. COM/2019/640 final. (2019). Recuperado de: https://eurlex.europa.eu/legal-content/ES/TXT/?uri=CELEX:52019DC0640.

En route to the Knowledge-Based Bio-Economy in Europe (2007). German Presidency of the European Union. Cologne. Recuperado de: https://dechema.de/dechema media/Downloads/Positionspapiere/Col ogne Paper.pdf. 
European Commission (2002). Life sciences and biotechnology, a strategy for Europe. Communication from the Commission to the European parliament, the Council, the Council, the Social and Economic Committee and the Committee of the Regions. Recuperado de: https://eur-

lex.europa.eu/\%20LexUriServ/LexUriServ.do?uri=COM:2002:0027:FIN:EN:PD F.

European Commission (2012). Innovating for sustainable growth. A bioeconomy for Europe. Recuperado de: https://ec.europa.eu/research/bioeconomy/pdf/officialstrategy_en.pdf.

European Commission. (2018). A sustainable bioeconomy for Europe: strengthening the connection between economy, society and the environment. Updated bioeconomy strategy. Recuperado de: https://ec.europa.eu/research/bioeconomy/pdf/ec bioeconomy strate gy 2018.pdf.

European Commission. (2020). World Bioeconomy Forum 2020. Recuperado de: https://ec.europa.eu/irc/en/event/conference/world-bioeconomyforum-2020.

European Commission. International ocean governance: an agenda for the future of our oceans. (2019). Recuperado de: https://ec.europa.eu/maritimeaffairs/policy/ocean-governance en.

European Commission, Food 2030. (2015). Recuperado de: https://ec.europa.eu/info/research-and-innovation/researcharea/bioeconomy/food-2030 es.

International Advisory Council on Global Bioeconomy, IACGB. (2020). Expanding the Sustainable Bioeconomy - Vision and Way Forward. Communiqué of the Global Bioeconomy Summit 2020. Recuperado de: https://gbs2020.net/wp-content/uploads/2020/11/GBS2020 IACGB-

Communique.pdf. 
International Advisory Council on Global Bioeconomy, IACGB. (2020). Global Bioeconomy Policy Report (IV): A decade of bioeconomy policy development around the world. Recuperado de: https://gbs2020.net/wp-content/uploads/2020/11/GBS-2020_GlobalBioeconomy-Policy-Report_IV_web.pdf.

MARVIK, O.J., PHILP, J. (2020). The systemic challenge of the bioeconomy. A policy framework for transitioning towards a sustainable carbon cycle. EMBO Reports, 21 (10), 1-6. https://doi.org/10.15252/embr.202051478.

MENGAL, Ph., WUBBOlTS, M., ZICA, E., RUIZ, A, BRIGITT, D., PIENIA, A. \& BLACK, S. (2018). Bio-based Industries Joint Undertaking: The catalyst for sustainable bio-based economic growth in Europe. New Biotechnology, 40 Part A, 31 39. https://doi.org/10.1016/i.nbt.2017.06.002.

National Bioeconomy Blueprint (2012). The White House. Washington. Recuperado de: https://obamawhitehouse.archives.gov/sites/default/files/microsites/ostp /national_bioeconomy_blueprint_april_2012.pdf.

PATERMANN, C. \& AGUILAR, A. (2018). The origins of the bioeconomy in the European Union. New Biotechnology, 40 Part A, 20-24. https://doi.org/10.1016/j.nbt.2017.04.002.

Reglamento (UE) $n^{\circ} 560 / 2014$ del Consejo, de 6 de mayo de 2014, por el que se establece la Empresa Común para las Bioindustrias. Diario Oficial de la Unión Europea. L 169/130, 7.6.2014, p. 130-151. Recuperado de: https://eur-lex.europa.eu/legalcontent/ES/TXT/PDF/?uri=CELEX:32014R0560\&from=ES.

RUIZ SIERRA, A., ZICA, E., LANGE, L., LLORENTE RUIZ DE ARZÚA., P., CANALISA, A., MALLORQUÍN ESTEBAN, P., PAIANO., P. \& MENGAL, Ph. (2020). The biobased industries joint undertaking: A high impact initiative that is transforming the bio-based industries in Europe. New Biotechnology, 60, 105-112. https://doi.org/10.1016/j.nbt.2020.09.003. 
The Bioeconomy to 2030: designing a policy agenda. (2009). OECD. Recuperado de:

https://www.oecd.org/futures/longtermtechnologicalsocietalchallenges/thebioeconomyto2030designinga policyagenda.htm.

ULRICH VON WEIZSÄKER, E. \& WIJKMAN, A. (2018). Come on! Capitalism, shorttermism, population, and the destruction of the planet. Club of Rome. New York, Springer.

WOHLGEMUTH, R., TWARDOWSKI, T. \& AGUILAR, A. (2021). Bioeconomy Moving Forward Step by Step - A Global Journey. New Biotechnology, 61, 22-28. https://doi.org/10.1016/j.nbt.2020.11.006. 